\title{
Modelling charge storage near full well in CCDs
}

\author{
D.P. Weatherill, ${ }^{1}$ R. Plackett, K. Arndt and I.P.J. Shipsey \\ University of Oxford, \\ Keble Road, Oxford OX1 3RH, U.K. \\ E-mail: daniel.weatherill@physics.ox.ac.uk
}

AвSTRACT: The shape and size of the stored charge packet within a CCD pixel after exposure has implications for optimal device operation, susceptibility to radiation damage and modelling of dynamic charge collection effects such as the "Brighter-Fatter Effect". Above the full well capacity, phenomena such as bleed trails and surface charge loss occur. In this paper we discuss why accurately reproducing saturation effects in simulations based on electrostatics is difficult, and present an approach to modelling the storage of charge in CCD pixels using commercial semiconductor simulation software. We suggest experimental measurements which can be connected to such modelling. Full well measurements on a thick, high resistivity back illuminated sensor (the e2v CCD261) are presented.

KEYwords: Detector modelling and simulations II (electric fields, charge transport, multiplication and induction, pulse formation, electron emission, etc); Detectors for UV, visible and IR photons

${ }^{1}$ Corresponding author. 


\section{Contents}

1 Introduction 1

2 Background 1

2.1 Charge storage 1

2.2 Full well 3

3 TCAD simulation method $\quad 4$

4 CCD261 measurements $\quad 7$

$\begin{array}{lll}4.1 & \text { Estimation of device parameters } & 7\end{array}$

4.2 Full well measurement using TDI transfer curve 9

5 Summary and conclusion $\quad 10$

\section{Introduction}

The spatial distribution of collected charge within the potential well of a CCD pixel has been of interest since the beginning of the device's use in imaging [1]. For space-borne applications where induced charge transfer inefficiency due to radiation damage is important, a model for the storage volume may be used to estimate the number of traps which will be "seen" by a given charge packet as it transfers through the array. Notably, such an approach (based on a simple power law relationship between signal size in electrons and occupied volume) is used in CTI correction for the Gaia mission [2]. Detailed CTI measurements on Gaia test devices later supported the validity of this approximation [3]. Determination of individual trap energy levels from "trap-pumping" data is also possible in conjunction with a model for stored charge density [4]. Investigations of correlated charge collection effects in thick, fully depleted sensors (i.e. the "Brighter-Fatter Effect") have shown that the change in effective storage depth as signal charge accumulates theoretically contributes to the magnitude of correlation [5]. In this context, the change in storage depth is closely connected to the relationship between signal size and storage volume.

Calculation of the volume of a signal packet in a CCD using numerical TCAD software has been previously presented [6], using models constructed from device design parameters. In this contribution, we present results using the same technique but estimating the required parameters from electrooptical measurements, and show good agreement with full well capacity measurements of the device.

\section{Background}

\subsection{Charge storage}

Throughout the following discussion, an n-channel CCD is assumed. However, the same arguments should apply equally to a p-channel device. We also assume a fully depleted device. In this idealised 


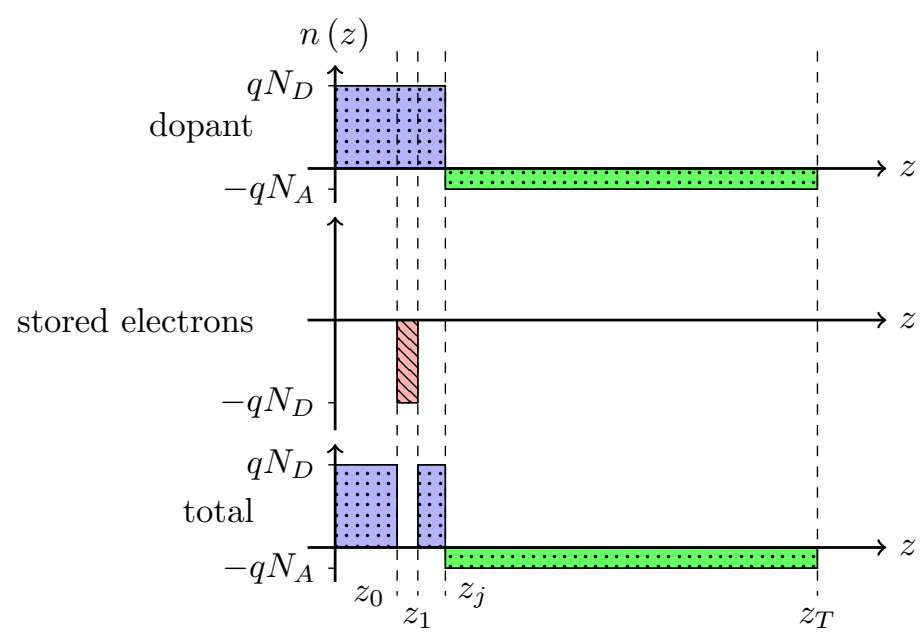

Figure 1. Schematic representation of pixel charge storage in 1D.

situation, recombination due to bulk traps is also neglected, as is generation due to dark current, which is highly suppressed at the typical operating temperature of a scientific CCD. Consider a CCD with energised collecting gates, which has been depleted by clocking the array and emptying charge through the reset drain in the usual manner: the electron density $n$ in the buried channel is very low, and all the donor impurities are ionised. As photo-generated electrons arrive and are stored, $n$ increases. The maximum possible density is $n=N_{D}$, i.e. where local charge neutrality has been reached. One possible simplifying assumption is that this condition applies at all times, in which case in 1D an exact formula for the potential in a CCD including stored charge can be found by solving Poisson's equation [7]. The charge density through depth in this scenario is illustrated in figure 1. However, it is not clear that the assumption is always a good one, and we now turn to the implications of its relaxation.

For a potential $\psi$, the electron density in a semiconductor is given by [8, eq $2.25 \mathrm{a}]$ :

$$
n=n_{i} \cdot \exp \left(\bar{\psi}-\bar{\phi}_{n}\right)
$$

where $n_{i}$ is the intrinsic concentration, $\phi_{n}$ is the electron quasi-fermi level, and the overbar denotes scaling by the thermal voltage, i.e. $\bar{\psi}=\frac{q \psi}{k_{B} T}$, where $q$ is the electronic charge, $k_{B}$ is Boltzmann's constant, and $T$ is the absolute temperature. In a buried channel CCD, the value of $\phi_{n}$ within the channel is determined by the reset drain voltage and the gate voltage (for a very detailed discussion see $[9, \S 1.4 .3 .1])$ Using the drift-diffusion equation for electrons:

$$
\begin{aligned}
& \mathbf{J}_{n}=-n \mu_{n} k_{B} T \nabla \bar{\psi}+q D_{n} \nabla n \\
\Rightarrow & \mathbf{J}_{n}=-n \mu_{n} k_{B} T \nabla \bar{\psi}+q D_{n} n \nabla(\bar{\psi}-\bar{\phi})
\end{aligned}
$$

where $\mathbf{J}_{n}$ is the electron current density, $\mu_{n}$ is the electron mobility and $D_{n}$ is the electron diffusivity; and assuming the Einstein relation $q D_{n}=\mu_{n} k_{B} T$ applies, we obtain:

$$
\mathbf{J}_{n}=-n \mu_{n} k_{B} T \nabla \bar{\phi}
$$


If the stored charge distributions are static in time (i.e. $\mathbf{J}_{n}=0$ ), then (2.4) implies that $\phi_{n}$ is spatially constant. (2.1) then leads to:

$$
n=n_{\max } \exp \left(\bar{\psi}-\bar{\psi}_{\max }\right)
$$

where $n_{\max }$ is the maximum charge density and $\bar{\psi}_{\max }$ is the channel potential maximum. Within the buried channel, we can then write Poisson's equation as:

$$
\nabla \cdot(\varepsilon \nabla \psi)=n_{\max } \exp \left(\bar{\psi}-\bar{\psi}_{\max }\right)-N_{D}
$$

where for simplicity we have assumed the minority (hole) concentration is zero. The non-linearity of (2.6) is the source of difficulty in accurately simulating charge storage using a simple electrostatic solver. It should be noted that limits on the bounds of this inaccuracy have been investigated quantitatively by Dale [10] and found to be insignificant for many purposes. Analytical solutions to (2.6) are not possible, but some points about the shape of the stored charge can be determined (a related analysis was performed by El-Sissi and Cobbold [11]). At the exact position of the potential maximum (i.e. $\bar{\psi}-\bar{\psi}_{\max }=0$ ), (2.6) reduces to the linear case. However, the further the distance from the potential maximum, the worse the linear approximation becomes.

\subsection{Full well}

The term "Full Well Capacity" refers to the maximum useful quantity of charge which can be stored in a CCD pixel. Broadly speaking, there are two physical mechanisms by which full well can be exceeded, both of which are schematically illustrated in figure 2 :

1. As electrons accumulate in the buried channel storage region, the potential under the collecting phase decreases (the empty collecting phase is represented by the solid blue line in figure 2). When the potential reaches the same level as that under a neighbouring barrier phase (solid red line in figure 2), stored electrons are no longer confined by the barrier phase and may travel up and down a column, resulting in a bleed trail. Following the terminology of Janesick [9], this condition is referred to as "bloomed full well", and is shown by the blue dotted line in figure 2. The signal level at which blooming occurs can be increased by increasing the collecting phase gate potential relative to that of the barrier phase.

2. As stored charge accumulates, the shape of the confining potential in depth changes, becoming "flatter" at the well. Thus the stored charge packet increases in thickness towards the oxide interface. If the collecting phase gate potential is sufficiently high, the charge packet may contact the oxide interface before bloomed full well is reached. This situation is represented by the dashed line in figure 2 . Contact with the surface results in recombination with free holes at the interface or deferral of signal charge due to surface trapping states [9], causing signal loss or Charge Transfer Inefficiency. This is known as "surface full well".

In operation, it is often preferential to tune the $\mathrm{CCD}$ for maximum full well, that is set the clock voltages such that blooming full well and surface full well occur at the same signal level. In other cases, the charge loss and noise implications of surface full well are unacceptable, so the device might be operated slightly below the maximum possible full well capability [9].

Using the definitions given above, it is possible to estimate both bloomed full well and surface full well points from the linear Poisson solution given by Yin and Cooper [7]. 


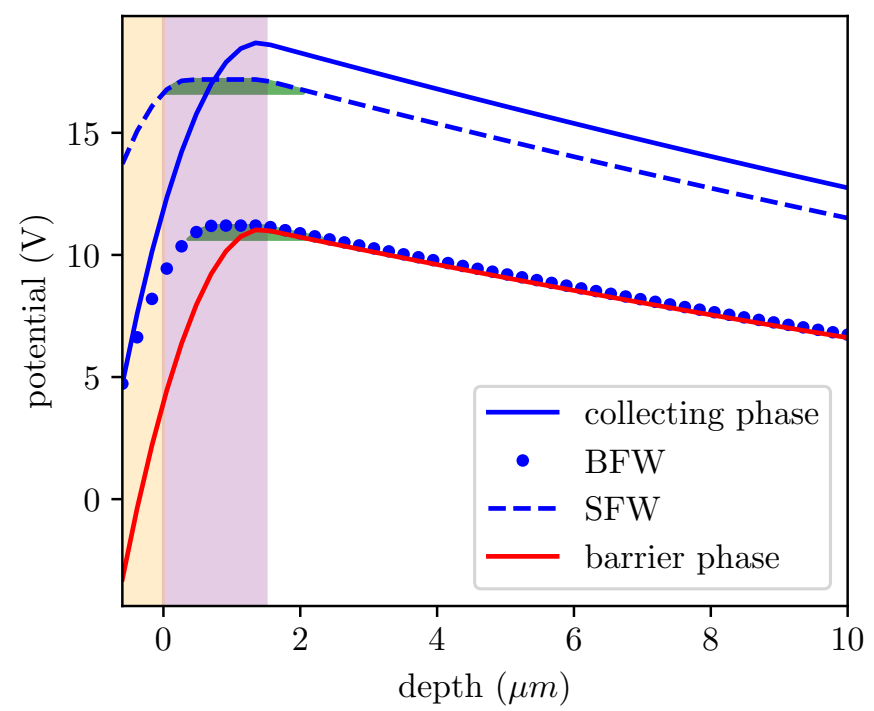

Figure 2. Schematic diagram showing potential structure for full well conditions. The orange shaded region represents the oxide layer, and the purple shaded region the buried channel implant.

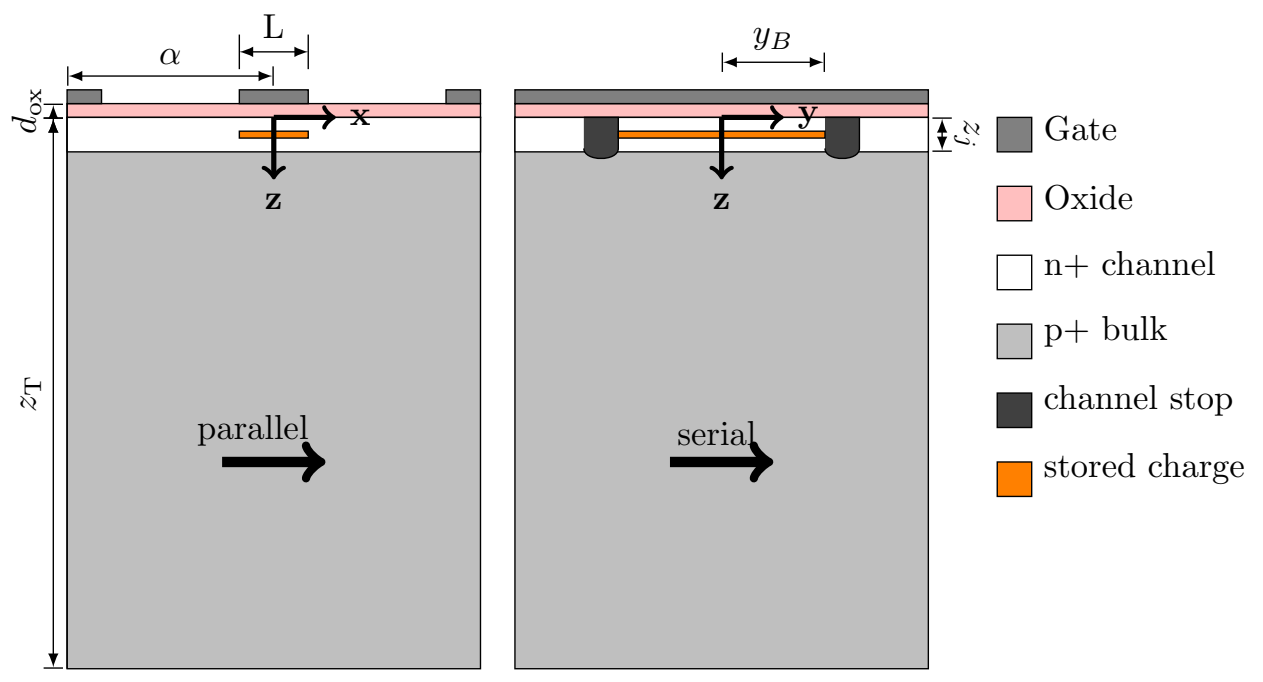

Figure 3. CCD geometry used in TCAD simulations.

\section{TCAD simulation method}

The commercially available Silvaco ATLAS semiconductor device simulator software [12] was used to construct a 2D CCD pixel model, whose geometry is representative of the e2v CCD261 (for which some experimental results are shown in section 4). Perfectly planar geometry is assumed; no process simulation was performed. A diagram of the geometry is shown in figure 3. The gate oxide (which in reality consists of an $\mathrm{SiO}_{2} / \mathrm{Si}_{3} \mathrm{~N}_{4}$ stackup) was modelled as a single layer of $\mathrm{SiO}_{2}$ 
Table 1. Geometry and material parameters used in TCAD model.

\begin{tabular}{|r|r|r|}
\hline Parameter & value & description \\
\hline $\mathrm{L}$ & $5 \mu \mathrm{m}$ & collecting gate width \\
$\alpha$ & $15 \mu \mathrm{m}$ & pixel pitch \\
$d_{\mathrm{Ox}}$ & $0.15 \mu \mathrm{m}$ & effective gate thickness \\
$z_{T}$ & $100 \mu \mathrm{m}$ & device thickness \\
$z_{j}$ & $1.8 \mu \mathrm{m}$ & junction depth \\
$y_{B}$ & $7 \mu \mathrm{m}$ & channel stop position \\
$N_{D}$ & $1.5 \times 10^{14} \mathrm{~cm}^{-3}$ & buried channel donor density \\
$N_{A}$ & $2 \times 10^{12} \mathrm{~cm}^{-3}$ & substrate acceptor density \\
$\varepsilon_{\mathrm{Ox}}$ & 3.4 & $\mathrm{SiO}_{2}$ permittivity \\
$\varepsilon_{\mathrm{Si}}$ & 11.7 & $\mathrm{Si}$ permittivity \\
$\varepsilon_{\mathrm{Ni}}$ & 7.0 & $\mathrm{Si}_{3} \mathrm{~N}_{4}$ permittivity \\
\hline
\end{tabular}

with an effective thickness. Values for the dopant concentrations and junction depth were estimated from electro-optical measurements (see subsection 4.1).

The charge storage simulation flow proceeds as follows:

- initialize a suitably fine mesh (sub Debye-length scale), and guess initial values for carrier concentrations and potential using local charge neutrality

- sweep applied gate voltage $V_{G}$ and back side bias $V_{B}$ to desired values, solving the Poisson equation only at each step

- artificially set the quasi fermi level $\phi_{n}$ throughout the mesh and perform a short transient simulation, which causes the pixel well to empty, leaving some remaining stored charge.

- solve the continuity equations for electrons and holes in steady state.

By varying the value of $\phi_{n}$ for the same values of $V_{G}$ and $V_{B}$, different amounts of stored charge can be investigated. Some example cutlines of the resulting potential and electron concentration from such simulations are shown in figure 4 . The phenomena of both blooming (charge density rapidly extending in the parallel direction) and surface contact are clearly visible at low quasi fermi levels. The full well characteristics are qualitatively even better observed in figure 5 .

Full well can be measured simply using cutlines at the edge of the pixel, and at the oxide interface. The onset of rapid increase in electron density found at these cutlines is a proxy for blooming full well and surface full well respectively. This process is illustrated in the left panel of figure 6. A cutline through the centre of the pixel can be used to calculate the centroid of the charge density, and thus find the effective storage depth (right panel of figure 6). The possible connection of the effective storage depth to measurable quantities is a subject for future work. Calculations were performed at a range of values for $V_{G}$ from $7.0 \mathrm{~V}$ to $10.0 \mathrm{~V}$, which are compared to experimental measurements in subsection 4.2. 

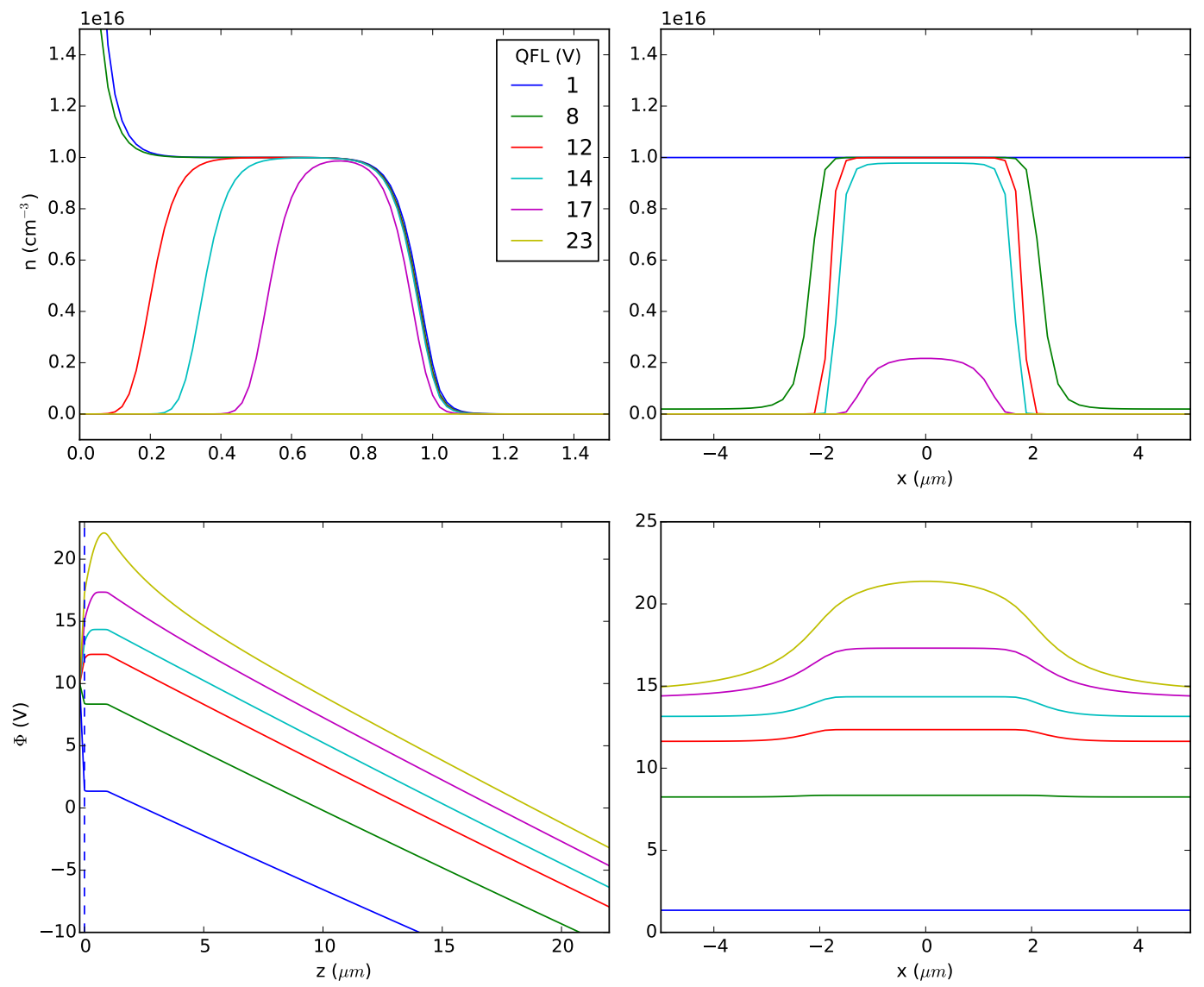

Figure 4. Selected cutlines through depth (left panels) and parallel transfer direction (right panels) from TCAD simulations with $V_{G}=10 \mathrm{~V}$ and $V_{B}=-60 \mathrm{~V}$ showing how potential (lower panels) and electron density (upper panels) change with the reset QFL.

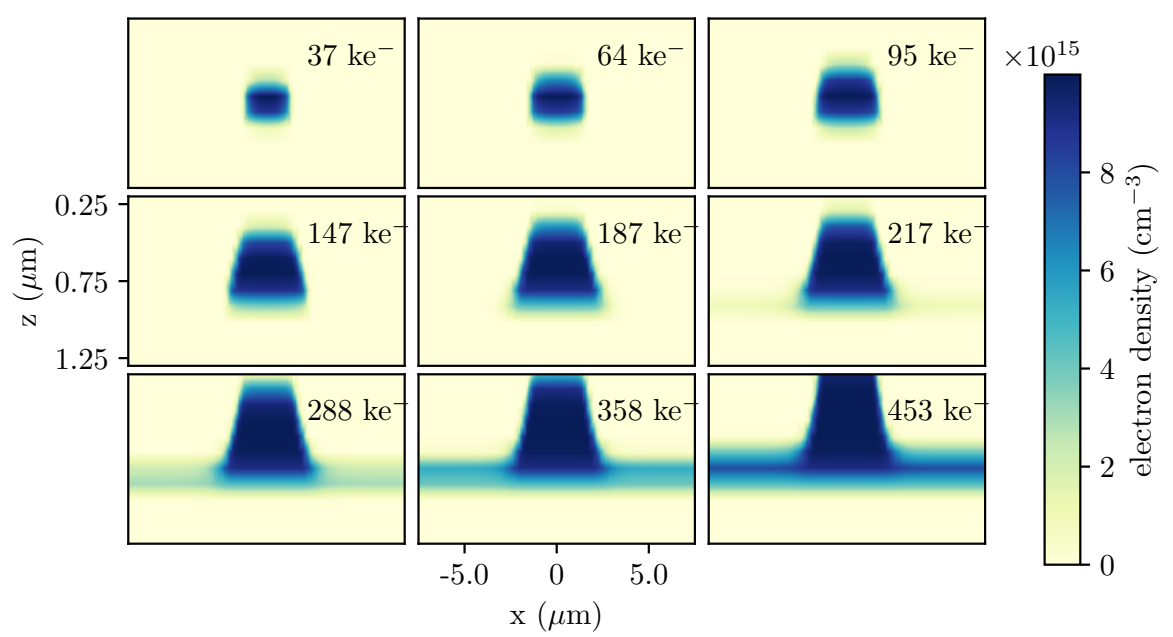

Figure 5. Plots showing the shape of the stored charge packet as more electrons are added. 

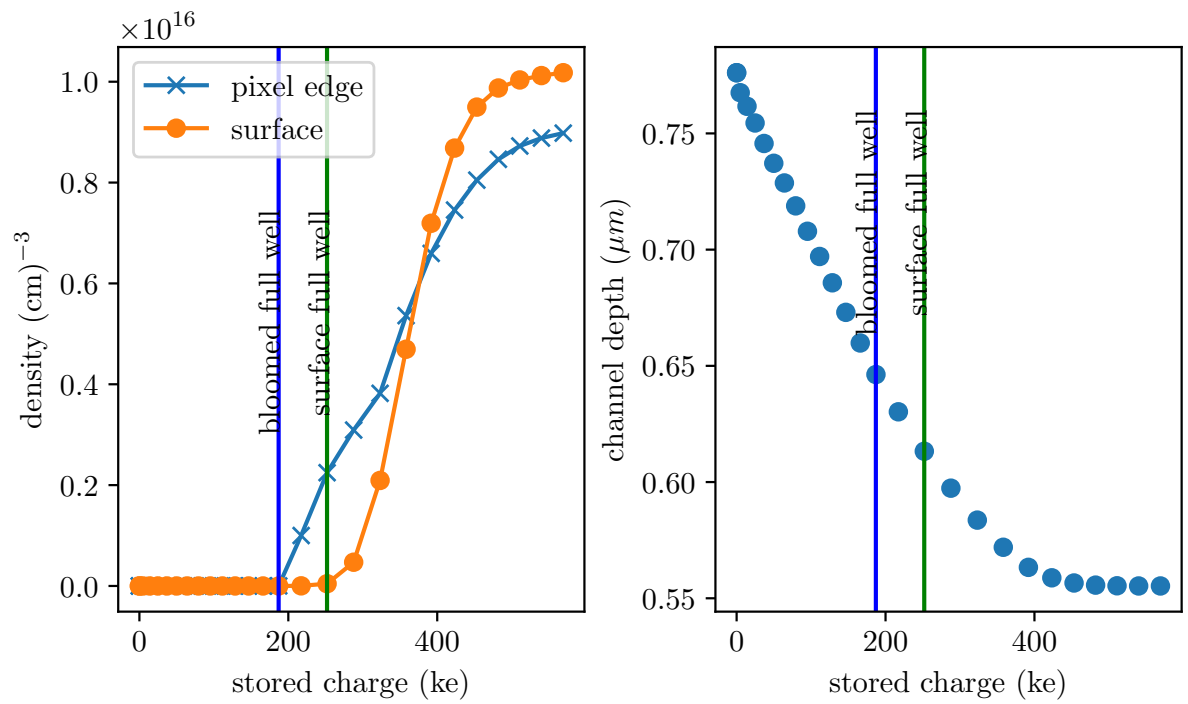

Figure 6. Finding the position of full well by observing the edge of the device mesh (left panel), and showing how the centroid channel depth moves towards the surface with increasing stored charge (right panel).

\section{CCD261 measurements}

The e2v CCD261 is a thick, back-illuminated device fabricated on high-resistivity bulk silicon [13]. Detailed electro-optical tests on this type of device have been previously presented by Robbins et al. [14]. Similar experiments and further investigation of correlated charge collection has been performed by Weatherill [15] on the exact device also used in this study.

\subsection{Estimation of device parameters}

The quantities which must be estimated to construct a TCAD model of the CCD261 are the two dopant densities $N_{D}$ and $N_{A}$ and the metallurgical junction depth $z_{j}$. These are obtained indirectly from measurements of substrate resistivity, channel potential and full well capacity. The "channel parameter" (i.e. the maximum potential in the channel when the gate voltage is $V_{G}=0$, but the buried channel has been depleted) $\psi_{\text {ch0 }}$ was measured by the usual method of finding the onset of charge injection at the output node (described in detail in [9, §1.4.3] and [15, §5.4.1]). For the particular device under test, it was found that $\psi_{\mathrm{ch} 0}=(9.11 \pm 0.04) \mathrm{V}$. Note that as mentioned by $[16, \S 3]$, to obtain true physical values for the channel parameter in the imaging array from the measurement of channel parameter using the method of injecting charge into the output transistor, one would need to know (or simulate) the flat-band voltage characteristics of that transistor. In fact, since the free parameters in the TCAD simulation are $z_{J}, N_{A}, N_{D}$ and $d_{\mathrm{ox}}$, the last of which is unknown, we manually adjust the value of $d_{\mathrm{ox}}$ in the TCAD simulation (with $z_{T}$ fixed and our fitted electro-optical values for all other parameters) until the channel maximum observed in the simulation agrees with that observed in measurements to $\approx 0.5 \mathrm{~V}$. The errors introduced from not knowing the value of $d_{\mathrm{ox}}$, not using a realistic doping profile in the simulation, and not knowing the flat-band voltage of the output transistor likely constitute most of the error in the resulting predictions of full well value.

Next the resistivity of the silicon was estimated following the technique described by Holland et al. [17]: a small, focussed spot of light is projected onto the CCD and the spot radius is measured 


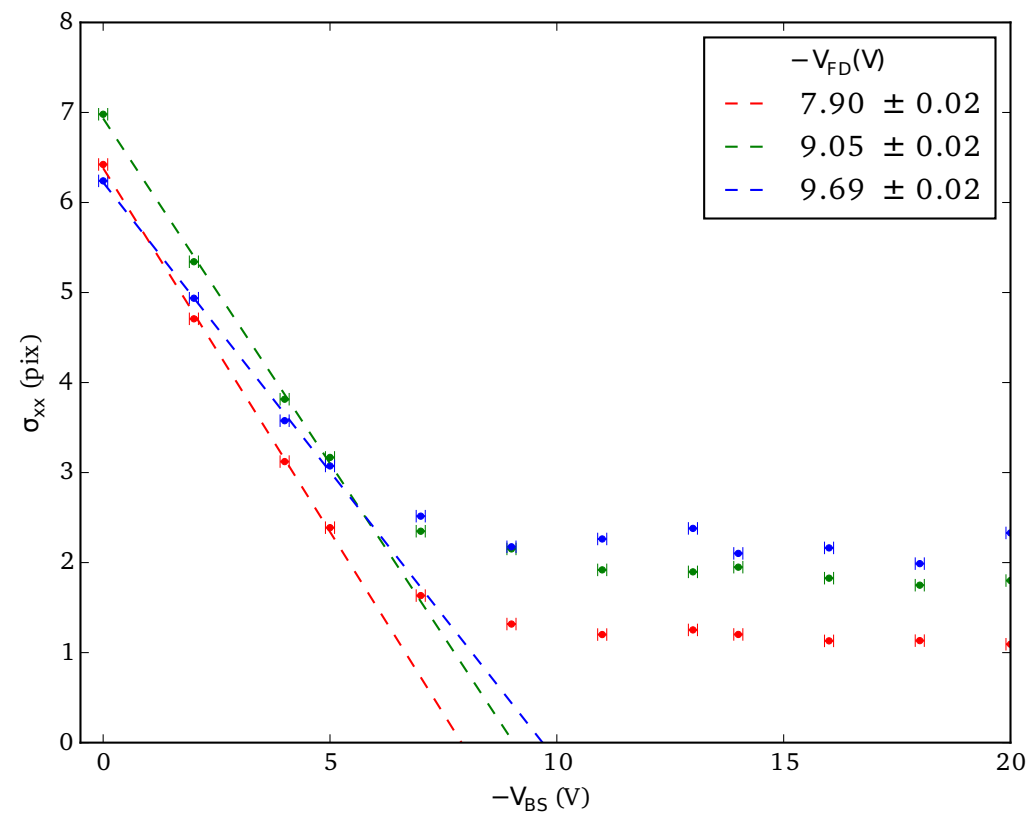

Figure 7. Determination of the full depletion voltage by measuring the width of a spot $\sigma$ against back bias voltage $V_{B}$ for 3 wavelengths: $650 \mathrm{~nm}$ (red), $525 \mathrm{~nm}$ (green) and $470 \mathrm{~nm}$ (blue).

as the back side bias is adjusted. When a field free region is present in the device (low bias), the width of the spot is large due to diffusion in this region. A "knee point" in the curve is observed as the device reaches full depletion and diffusion is drastically reduced. The experimental results are shown in figure 7 . The full depletion voltage is consistent with the measurement performed by Robbins et al. [14] on a similar device. The substrate doping is then estimated using the equation from Fairfield et al. [18, eq. 3]:

$$
V_{B}=V_{G}+\psi_{\mathrm{ch}}+\frac{q N_{A}}{2 \varepsilon_{\mathrm{Si}} \varepsilon_{0}}\left(z_{T}-\sigma\right)^{2}
$$

where $\sigma$ is the measured diffusion width of the spot. Finally, we estimate $N_{D}$ and $z_{J}$ using a simultaneous fit to the linear Poisson solution of Yin and Cooper [7]. For detailed discussion on this fitting procedure, see Weatherill $[15, \S 6.2]$. It is noted that this rather simplistic method of estimating $N_{A}$ is likely to become less accurate with decreasing device thickness $z_{T}$, since there is significant 3D structure in the channel potential for quite some distance below the collecting gates, whereas using a single value in the estimating equation essentially assumes a 1D structure throughout the device. Indeed this possibility was noted in previous work using the method [18]. We consider, however, that the approximation is likely to be sufficient for the purpose at hand: for instance, it has been used to measure consistently the value of $z_{T}=150 \mu \mathrm{m}$ (see [14]). Here, we are using the approach only to find $N_{A}$, with $z_{T}=100 \mu \mathrm{m}$ a fixed value. The limits to using a 1D potential approximation in this analysis has not, to our knowledge, been fully examined in the literature, though some related comments are made by [16] in connection with a much thinner $\left(z_{T} \approx 40 \mu \mathrm{m}\right)$, though also not back-biased, device. In that work, the authors compared 


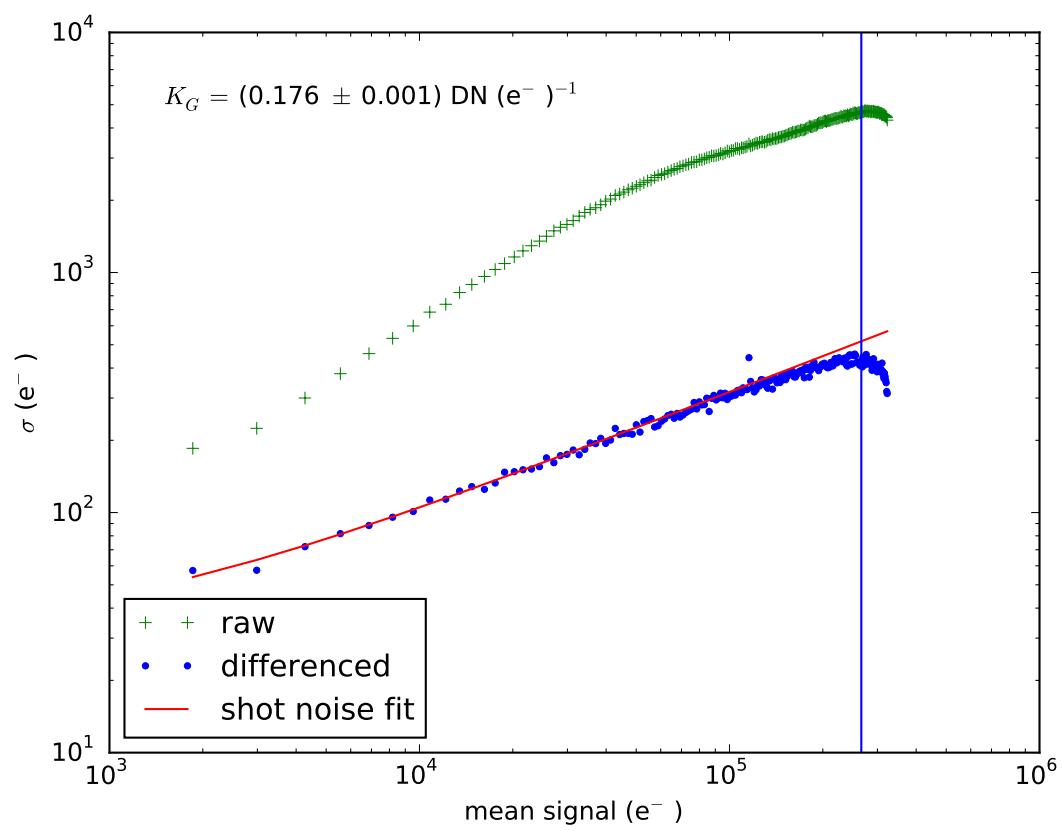

Figure 8. Example TDI photon transfer curve from CCD261, operated at $V_{G}=9.27 \mathrm{~V}, V_{B}=-60.0 \mathrm{~V}$. The vertical blue line indicates the fitted full well capacity $Q_{F W}=240 \mathrm{ke}^{-}$.

a 1D potential model to a 2D finite element simulation, calculating potential distributions from doping (rather than the inverse problem essentially described here), and found that the resulting "measurable" channel potential maximum changed by $\approx 0.6 \mathrm{~V}$, and the depth of the maximum by $\approx 0.2 \mu m([16$, figure 4]).

\subsection{Full well measurement using TDI transfer curve}

A TDI transfer curve method was used to measure the full well behaviour with varying applied gate potential $V_{G}$ and back side bias $V_{B}$. The device is clocked out continuously whilst illuminated with a flat field source, with a time delay inserted in the timing sequence between each parallel transfer. The statistics for each row of data then form a mean-variance data set which can be used for photon transfer analysis. Like a conventional PTC, a differenced frame pair is used to calculate the variance to eliminate fixed pattern noise. In the TDI transfer curve, though, the fixed pattern noise appears at low signal and is suppressed at high signal due to the spatial averaging over columns which occurs as more transfers happen. An example photon transfer curve is shown in figure 8 . Care must be taken to mask bad columns in the TDI data, since the statistical power is much lower than the equivalent full frame PTC, and a single extreme pixel value will slightly affect all values in subsequent rows of the same column. The principal advantage is that the voltage parameter space can be explored very rapidly, since only two images are required for each parameter set.

In agreement with the results of Robbins et al. [14], no change in full well capacity as measured in electrons is observed with changing only $V_{B}$, though the gain of the output does change. In subsection 4.2 the experimental measurements are shown along with results of the TCAD simulation 


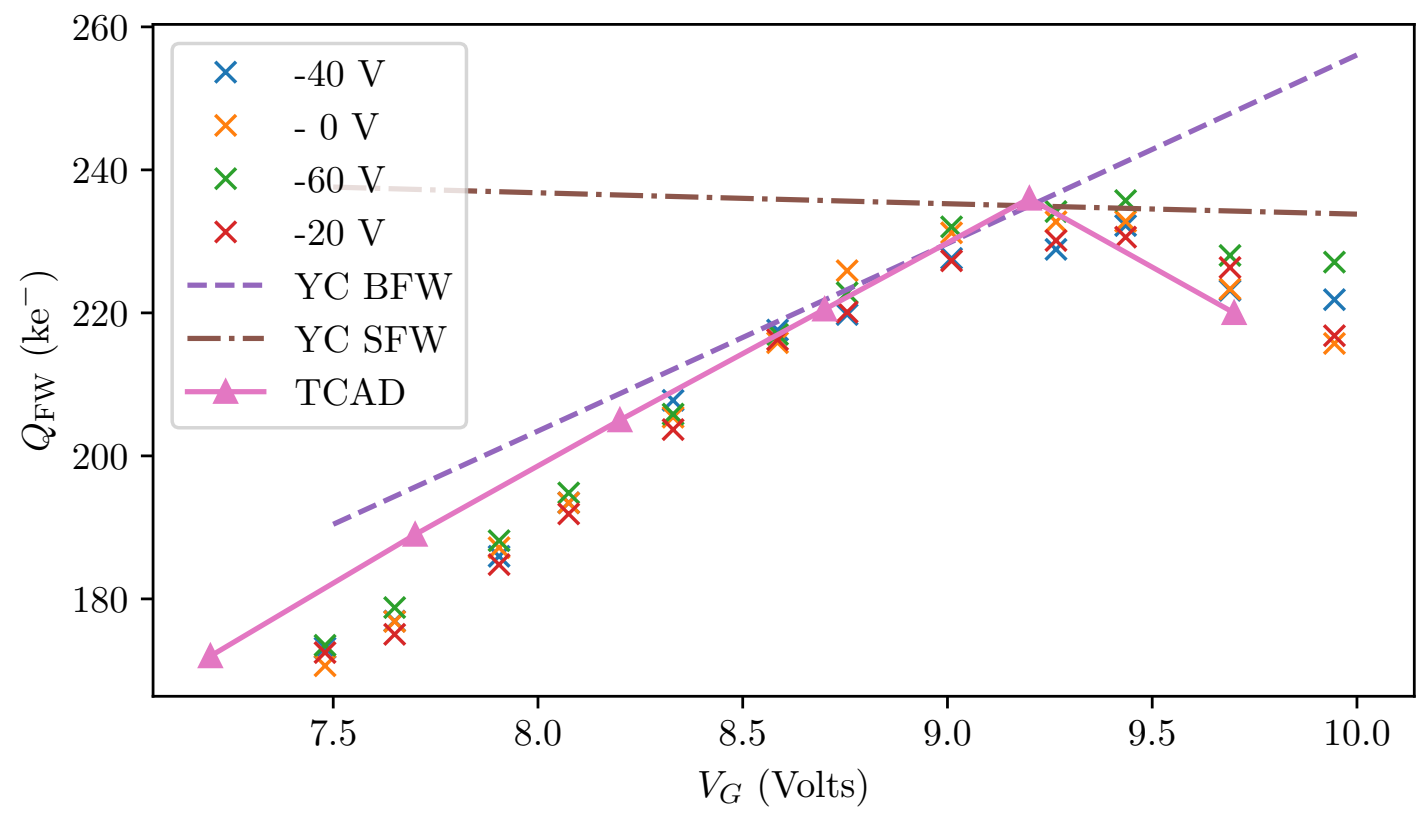

Figure 9. Measured and simulated full well capacities against collecting gate voltage. Crosses show experimental data for different back bias voltages, the dashed and dash-dot lines show estimates of bloomed full well and surface full well using the Yin and Cooper model, and the solid line with triangles shows TCAD calculation results.

method described in section 3, and estimated bloomed full well and surface full well values from Yin and Cooper's model [7]. Both the linear estimate and the TCAD results are shown to be in reasonable agreement with experiment, though at this stage no detailed sensitivity analysis of the TCAD method has been performed, so the possible extent of disagreement is not precisely known. Both the linear approximation and the TCAD method are found to reproduce the position of the optimum value of $V_{G}$ well.

\section{Summary and conclusion}

A method for modelling the storage of charge in CCD pixels using numerical TCAD software has been presented. It has been shown how device parameter values for the simulation can be obtained from simple electro-optical measurements on a thick, back biased CCD. The simulation method, applied to an e2v CCD261 was shown to give broad agreement with measurements of the full well capacity against varying gate voltage.

We intend to pursue the development of this technique further in future work, using improved electro optical measurements on different CCD devices, detailed sensitivity analysis of the TCAD simulations, and a thorough comparison with various available approximate models for charge density and potential. It is also hoped that we may use this method to cross-validate results of simulations for correlated charge collection. 


\section{Acknowledgments}

The authors would like to acknowledge useful and illuminating discussions with Craig Lage (UC Davis); Andrew Rasmussen (SLAC); Paul O'Connor, Andrei Nomerotski and Merlin Fisher-Levine (BNL). We thank Konstantin Stefanov (The Open University) for assistance with Silvaco and Neil Murray (Dynamic Imaging Analytics) for help with running the CCD261.

\section{References}

[1] H.W. Hanneman and L.J.M. Esser, Field and potential distributions in charge-transfer devices, Philips Res. Rep. 30 (1975) 56.

[2] A. Short, C. Crowley, J.H.J. de Bruijne and T. Prod'homme, An analytical model of radiation-induced charge transfer inefficiency for CCD detectors, Mon. Not. Roy. Astron. Soc. 430 (2013) 3078 [arXiv: 1302 . 1416].

[3] G.R. Hopkinson, A. Short, C. Vetel, I. Zayer and A.D. Holland, Radiation effects on astrometric ccds at low operating temperatures, IEEE Trans. Nucl. Sci. 52 (2005) 2664.

[4] D.J. Hall, N.J. Murray, A.D. Holland, J. Gow, A. Clarke and D. Burt, Determination of in situ trap properties in CCDs using a "single-trap pumping” technique, IEEE Trans. Nucl. Sci. 61 (2014) 1826.

[5] A. Rasmussen et al., High fidelity point-spread function retrieval in the presence of electrostatic, hysteretic pixel response, Proc. SPIE 9915 (2016) 99151A.

[6] A.S. Clarke, D.J. Hall, A. Holland and D. Burt, Modelling charge storage in Euclid CCD structures, 2012 JINST 7 C01058.

[7] Y. Yin and J.A. Cooper Jr., Simple equations for the electrostatic potential in buried-channel MOS devices, IEEE Trans. Electron Dev. 39 (1992) 1770.

[8] S.M. Sze, Physics of semiconductor devices, Wiley-interscience, U.S.A., (2006).

[9] J.R. Janesick, Scientific charge-coupled devices, SPIE Press, Bellingham WA U.S.A., (2001).

[10] B. Dale, The validity of the depletion approximation applied to a bulk channel charge-coupled device, IEEE J. Solid-State Circ. 11 (1976) 207.

[11] H. El-Sissi and R.S.C. Cobbold, One-dimensional study of buried-channel charge-coupled devices, IEEE Trans. Electron Dev. 21 (1974) 437.

[12] Silvaco international, ATLAS user's manual, Santa Clara CA U.S.A., (2010).

[13] P.R. Jorden et al., Improving the red wavelength sensitivity of CCDs, Proc. SPIE 7742 (2010) $77420 \mathrm{~J}$.

[14] M.S. Robbins, P. Mistry and P.R. Jorden, Detailed characterisation of a new large area CCD manufactured on high resistivity silicon, Proc. SPIE 7875 (2011) 787507.

[15] D. Weatherill, Charge collection in silicon imaging sensors, Ph.D. thesis, The Open University, U.K., (2016).

[16] G.M. Seabroke, A.D. Holland, D. Burt and M.S. Robbins, Modelling electron distributions within ESA's Gaia satellite CCD pixels to mitigate radiation damage, Proc. SPIE 7439 (2009) 743905 [arXiv:0909.5116].

[17] S.E. Holland, D.E. Groom, N.P. Palaio, R.J. Stover and M. Wei, Fully depleted, back-illuminated charge-coupled devices fabricated on high-resistivity silicon, IEEE Trans. Electron Dev. 50 (2003) 225.

[18] J.A. Fairfield et al., Reduced charge diffusion in thick, fully depleted CCDs with enhanced red sensitivity, IEEE Trans. Nucl. Sci. 53 (2006) 3877. 